\title{
Defining Electricity Tariffs Using the Knowledge About the Consumers Profiles in ELECON Project
}

\author{
Hugo Morais, Zita Vale, Pedro Faria, Sérgio Ramos \\ GECAD - Knowledge Engineering and Decision Support Research Center \\ IPP - Polytechnic Institute of Porto \\ Porto, Portugal \\ hugvm@isep.ipp.pt, zav@isep.ipp.pt, pnfar@isep.ipp.pt, scr@isep.ipp.pt
}

\begin{abstract}
The increasing importance of the integration of distributed generation and demand response in the power systems operation and planning, namely at lower voltage levels of distribution networks and in the competitive environment of electricity markets, leads us to the concept of smart grids. In both traditional and smart grid operation, non-technical losses are a great economic concern, which can be addressed. In this context, the ELECON project addresses the use of demand response contributions to the identification of non-technical losses. The present paper proposes a methodology to be used by Virtual Power Players (VPPs), which are entities able to aggregate distributed small-size resources, aiming to define the best electricity tariffs for several, clusters of consumers. A case study based on real consumption data demonstrates the application of the proposed methodology.
\end{abstract}

Index Terms-Demand response, Elecon, electricity tariffs, nontechnical losses, smart grid.

\section{INTRODUCTION}

Future power systems will be characterized by the integration of several new concepts and paradigms, as the case of smart grids [1]; several resources, as the case of Distributed Generation (DG), and Demand Response (DR) [2]; and new players, as the case of Virtual Power Players (VPP), in the context of the increasingly competitive context.

The competitive environment, and the challenges that the operation and planning of power systems will face as well, will happen at lower levels of the power networks. This leads to the concerning of both generation (distributed generation) and demand-side response (demand response) and consumption resources of reduced power capacity, which require adequate players. This is the case of VPPs that aggregate distributed small-size resources in order to make them able to participate in the competitive context of electricity markets, supported by the smart grid infrastructure abilities [3].

An important concern in the power systems operation and planning is the existence of non-technical losses. These losses are due, for example, to the electricity theft by consumers. The unexpected load is verified in power system elements (lines, transformers, etc), making difficult to estimate the real system conditions in each moment, in addition to the obvious economic losses [4]. These losses gain increasing importance in the competitive environment of smart grid and electricity markets operation. Regarding the concepts and concerns mentioned above, and taking into account the cooperation between European and Brazilian partners, the ELECONElectricity Consumption Analysis to Promote Energy Efficiency Considering Demand Response and Non-technical Losses - project addresses the use of demand response contributions to identify non-technical losses.

In the methodology proposed in this paper, a Virtual Power Player (VPP) makes use of data mining techniques to classify the consumption data of several consumers, with the objective of determining the best electricity tariffs scheme for the consumers. These tariffs consider the time of the day, the day of the week, and the type of consumer. From the previous experience of the authors of the present paper, already presented in [5], the electricity tariffs will consider the electricity market prices and the context of VPP operation, giving a preliminary vision of the use of this methodology for the non-technical losses identification. This work is integrated in the work package WP2 of ELECON project having innovative and makes contributions in the following aspects:

- Definition of several distinct consumers' clusters, using data mining techniques, taking into account the consumption profiles;

- Definition of electricity tariffs by a VPP in its operation context, taking into account the consumers behavior;

- Use of real consumption data of several consumers in order to illustrate the proposed methodology.

The full paper, after the introductory section, will be structured as follows: Section II will briefly present the ELECON project; Section III will present the proposed electricity tariffs definition methodology; in Section IV, an illustrative case study will be presented; and, finally, Section $\mathrm{V}$ will present the main conclusions of the paper.

The research leading to these results has received funding from the People Programme (Marie Curie Actions) of the European Union's Seventh Framework Programme FP7/2007-2013/ under project ELECON - Electricity Consumption Analysis to Promote Energy Efficiency Considering Demand Response and Non-technical Losses, REA grant agreement No 318912.

This work is also supported by FEDER Funds through the "Programa Operacional Factores de Competitividade - COMPETE" program and by National Funds through FCT "Fundação para a Ciência e a Tecnologia" under the projects FCOMP-01-0124-FEDER: PEst-OE/EEI/UI0760/2011, PTDC/EEA-EEL/099832/2008, PTDC/SEN-ENR/099844/2008, and 


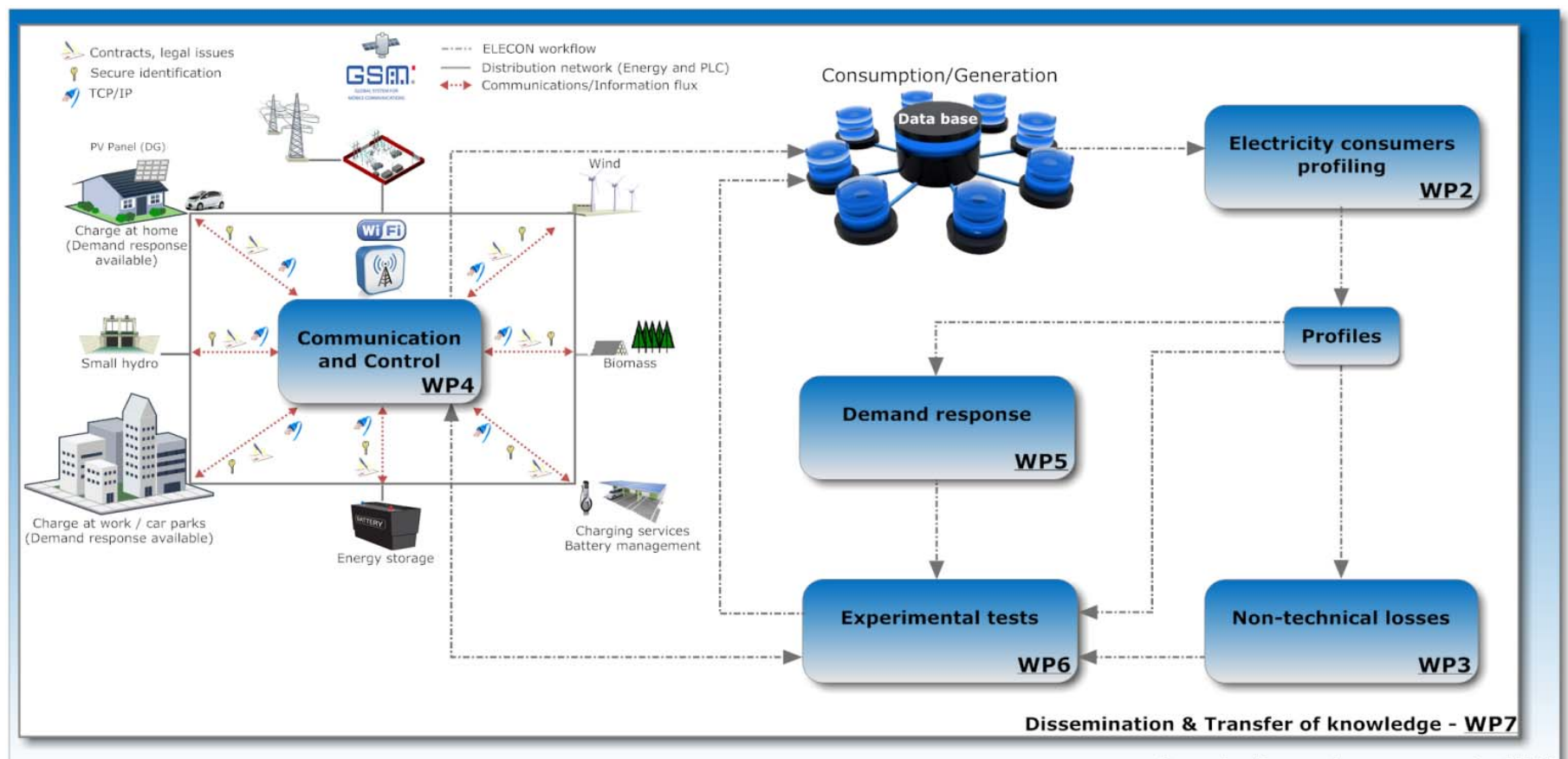

Organisation and management - WP1

Figure 1. ELECON project workflow.

\section{ELECON PROJECT - WORK PACKAGE 2}

The main goal of the ELECON project is to establish a competent and fruitful network between European and Brazilian researchers, whose research work contributes to the successful implementation of the smart grid concept. Fig. 1 presents ELECON project workflow.

The project aims to advance with electricity consumption analysis methods [6] and the way they are used to promote energy efficiency. This use is focused on the design and use of demand response programs and on the identification of nontechnical losses. On the one hand, if adequately used, demand response can play a crucial role for the sustainability of energy systems. On the other hand, the restructuring of the power industry business and the rapid evolution that is taking place towards real smart grids dramatically increases the number of players involved in electricity transactions, making billing much more complex. In the new context, the identification of non-technical losses assumes a new role and an increased importance in the scope of supervision procedures able to ensure the correctness of the billing process. The project also addresses the communication infrastructure and the innovative control models, which are required to bring these concepts to reality, considering adequate business models.

ELECON activities are organized in the following seven workpackages:

WP1 - Organisation and management

WP2 - Electricity consumers profiling

WP3 - Non-technical losses

WP4 - Communications and control in smart grids

WP5 - Demand response programs

WP6 - Experimental tests: Europe and Brazil benchmarking

WP7 - Dissemination \& Transfer of knowledge.
Work package WP2 focuses on the profiling of electricity consumers. Its main objectives are:

- To organize a library of electricity consumption data;

- To propose an electricity consumers profiling methodology;

- To undertake electricity consumer profiling and characterisation;

- To identify opportunities for efficiency improvement;

- To produce a report concerning the obtained electricity consumer profiles and the benchmarking studies.

This paper focuses in proposing consumption tariffs considering electricity consumers profiling methodology.

\section{CONSUMERS TARIFFS DEFINITION METHODOLOGY}

The main goal of the proposed methodology is to determine better tariffs schemes to be used by Virtual Power Players (VPP). VPPs are aggregator entities with the capacity of managing several energy resources such as a distributed generation, demand response programs, electric storage systems, electric vehicles and, in specific cases, the distribution network [7]. The main goal of the VPP is to guarantee its profits. However, the VPP needs to propose better conditions to the consumer than the traditional retailers (utilities).

In this away, the tariffs proposed by VPPs should be adapted to the consumption profile of each consumer and consider the acquisition energy cost by the VPP, considering the operation costs of the internal energy resources, the bilateral contracts and the electric market price forecast. The tariffs were determined by specific consumer's profiles in winter/summer and workdays/weekends. 
This division is important because the consumption profiles are very different in several types of consumers. For example, many industries sectors do not work in the weekend. On the other hand, the domestic consumers increase their demand in these periods. Figure 2 shows the proposed methodology used by the VPP to consumer's tariffs definition.

As one can see in Figure 2, the VPP tries to evaluate the impact of the tariffs' methodology, comparing the actual consumption with the historic ones. Using the tariffs impact it is possible to determine the consumption elasticity for each consumer and use this parameter to improve the consumer's profile.

\section{A. Consumers Clustering}

The division of consumers into clusters allows a better adoption of tariffs to each consumer, increasing the benefits of the VPP and consumers. One can define clustering as the process of partitioning a certain database into clusters based on a concept of similarity among data. Good clusters present objects with high similarity among them, and poor similarity among records of others clusters. There is a large variety of clustering algorithms that can be used, although there is no single algorithm that can discover by itself all sorts of cluster's shapes $[8,9]$.
Based on the Knowledge Discovery process applied to Databases (KDD) [10], a methodology to characterize the consumers has been implemented. Figure 4 shows the proposed methodology architecture, which includes the application of data mining techniques [11]

From the data acquisition, illustrated in Figure 4, a data pre-processing phase is required to detect and correct bad data ("clean the data"), and also a data-treatment to format data according to DM algorithms that will be used [5]. The required computational effort cannot be neglected, and whenever it is possible a reduction in the volume of database will be helpful. Typically, in consumers' load profile studies, the electrical costumers' consumption is represented by the typical daily load diagram and it can be imposed different load regimes, for example, for workdays and weekends or even for different seasons, resulting in the reduction of data for analysis, thus reducing the computational effort.

During this step, the normalization can be made to enable the comparison of the load diagram shape among the electrical consumers [12].

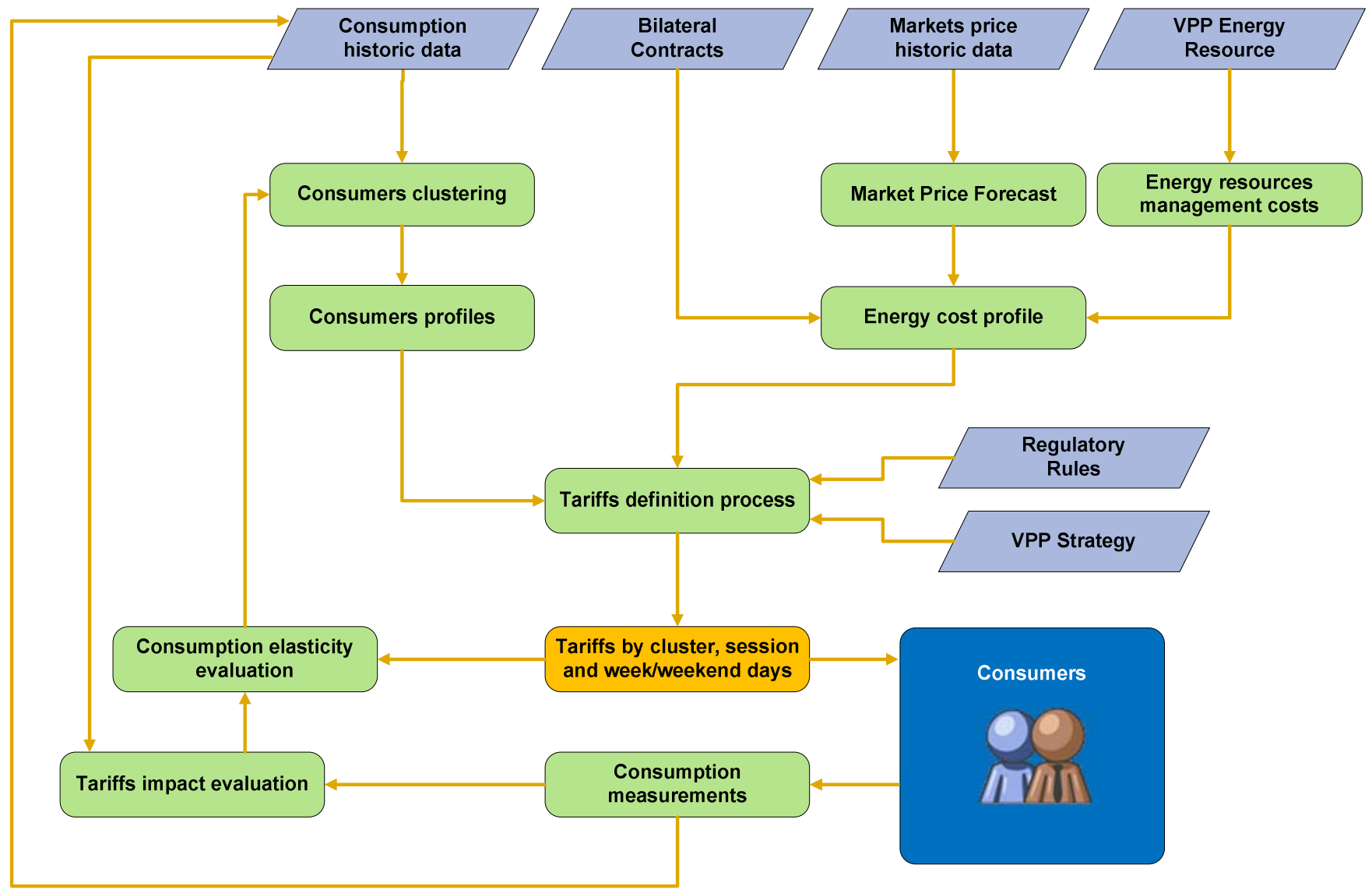

Figure 2. Tariffs definition framework. 


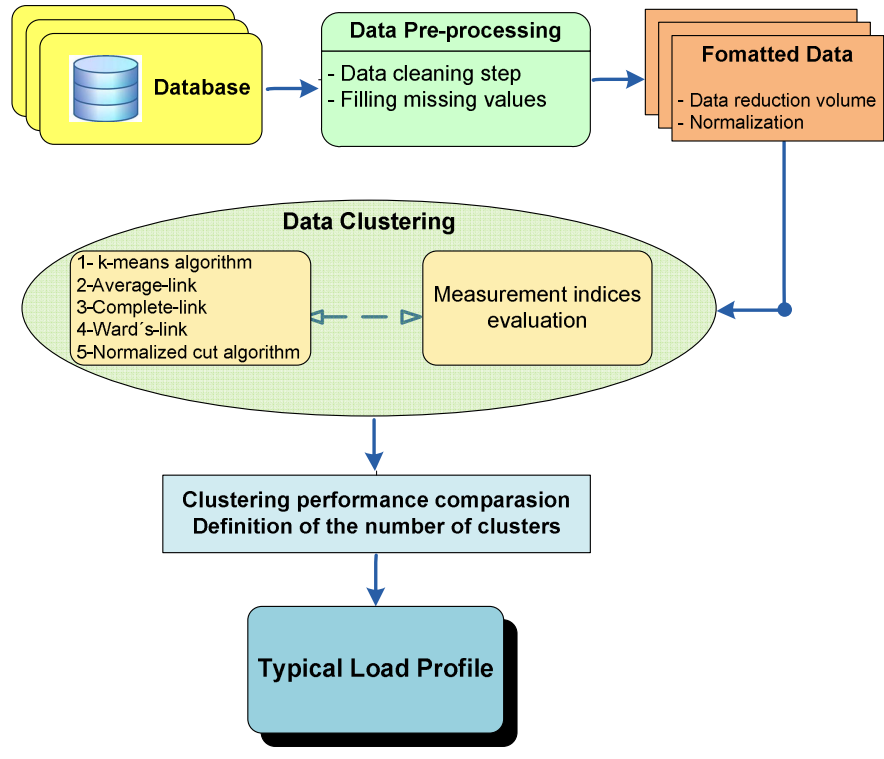

Figure 3. Clustering methodology architecture [5].

The implemented methodology proposes the use of several clustering algorithms in order to identify classes that are contained in the database. Clusters validity indices [9] can be used to test and evaluated the quality of the data partition, supporting the decision of the "best" number of clusters, as well as the choice of the number of clusters (in this step the experts' opinion is very important) that will be generated by the clustering algorithms. In the final step, the extracted knowledge can be validated (obtained load diagrams) including the interpretation and assessment of the data relations.

\section{B. Time-Of-Day periods definition}

An important aspect in the tariffs methodology is the definition of Time-Of-Day (TOD) periods. TOD is used to define different energy prices during the day inducing the response of consumers to these prices, changing their consumer profile. TOD pricing results from the classification of daily hours according to consumption homogeneity. In the proposed methodology, four TOD periods are considered: the peak hours; the half-peak hours; the off-peak hours; the super off-peak hours. These four TOD periods are selected based on the methodology used by the Portuguese Energy Sector Regulator Entity (ERSE) [13].

According to the consumption profile, ERSE defines different TOD for each voltage level. However, in practice, the consumers in Very-High Voltage (VHV), High Voltage (HV) and Medium Voltage (MV) have a similar consumption profile. Only the low voltage consumers present a different consumption profile. In Figure 4 are presented the consumption profiles in each voltage level (grid) in Portugal. However, for the VPPs each consumer should be analysed individually, according to their profile regardless the voltage level that is connected.

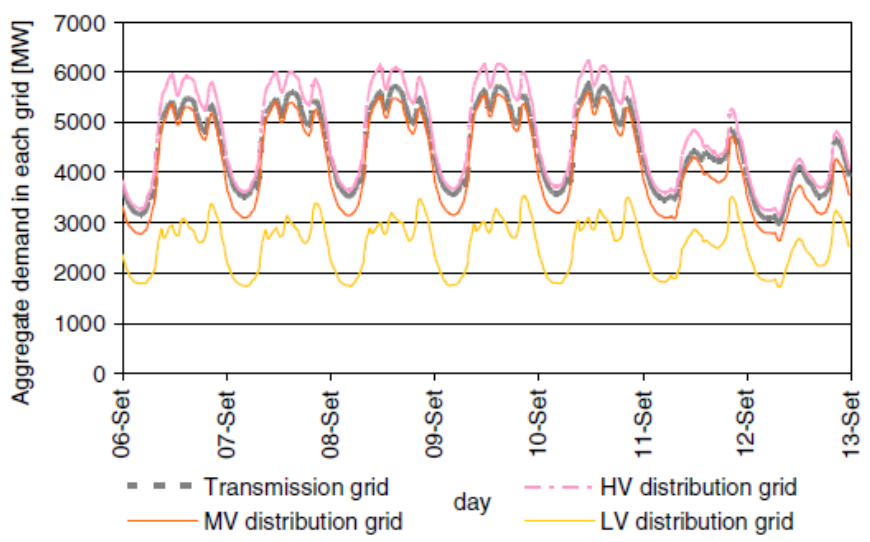

Figure 4. Aggregate demand profiles in several voltage level grids, in Portugal, for the second week of September, 2004 [13]

Other important aspect for the VPP's definition tariffs includes the regulatory rules. Theoretically, in liberalized electricity markets each aggregator or each retailer could define their tariffs freely. However, the regulatory entities impose several rules, like the number of hours in each TOD period. In Table I it is possible to see the number of hours in each TOD period imposed in Portugal by ERSE.

TABLE I. TIME-OF-DAY TARIFF PERIOD DURATION FOR WINTER AND SUMMER - WEEKLY-CYCLE**

\begin{tabular}{|c|c|c|c|}
\hline \multicolumn{2}{|c|}{ Winter } & \multicolumn{2}{|c|}{ Summer } \\
\hline Workdays & & Workdays & \\
\hline Peak & $5 \mathrm{~h} /$ day & Peak & $5 \mathrm{~h} /$ day \\
\hline Half-peak & $12 \mathrm{~h} /$ day & Half-peak & $12 \mathrm{~h} /$ day \\
\hline Normal off-peak & $3 \mathrm{~h} /$ day & Normal off-peak & $3 \mathrm{~h} / \mathrm{day}$ \\
\hline Super off-peak & $4 \mathrm{~h} /$ day & Super off-peak & $4 \mathrm{~h} /$ day \\
\hline Saturdays & & Saturdays & \\
\hline Half-peak & $7 \mathrm{~h} /$ day & Half-peak & $7 \mathrm{~h} /$ day \\
\hline Normal off-peak & 13h/day & Normal off-peak & 13h/day \\
\hline Super off-peak & $4 \mathrm{~h} /$ day & Super off-peak & $4 \mathrm{~h} /$ day \\
\hline Sundays & & Sundays & \\
\hline Normal off-peak & 20h/day & Normal off-peak & 20h/day \\
\hline Super off-peak & 4h/day & Super off-peak & 4h/day \\
\hline
\end{tabular}

In the proposed methodology the TOD periods are defined for each consumer considering the following objective function:

$$
f=\sqrt{\sum_{p} \sum_{i \in p}\left(\left(P_{(i, c)}+\alpha \frac{C_{(i, V P P)}}{C_{\mathrm{avg}(V P P)}}\right)-P_{(p, c)}\right)^{2}}
$$


Where:

\begin{tabular}{|c|c|}
\hline & $\begin{array}{l}\text { nction to minimize. It dep } \\
\text { e of tariff period } p\end{array}$ \\
\hline & $\operatorname{riod} p$ \\
\hline & $\begin{array}{l}\text { Tariff time period of peak hours (pk), half-peak } \\
\text { hours (hp), normal off-peak hours (nop) and super } \\
\text { off-peak hours (sop); }\end{array}$ \\
\hline 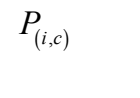 & $\begin{array}{l}\text { Average power demand, in the } 30 \text { minute time } \\
\text { interval } i \text {, of year } t \text {, for consumer profile } c \text {; }\end{array}$ \\
\hline$P_{(p, c)}$ & $\begin{array}{l}\text { Average power demand, in the tariff period } p \text {, of } \\
\text { year } t, \text { for consumer profile } c \text {; }\end{array}$ \\
\hline & VPP forecast energy acquisition cost in period $i$ \\
\hline$C_{\text {avg }(V P}$ & $\begin{array}{l}\text { VPP average forecast energy acquisition cost, of } \\
\text { year } t \text {; }\end{array}$ \\
\hline & Energy price impact factor \\
\hline
\end{tabular}

In TOD periods' definition VPP considers the consumption profile of each consumer $\left(P_{(i, c)}\right)$ for each 30 minutes and tries to minimize the difference between this consumption and the average power demand $\left(P_{(p, c)}\right)$ in TOD period $p$ for consumer profile $c$. The coefficient $C_{(i, V P P)} / C_{\text {avg(VPP) }}$ represents the cost of the energy acquisition in period $i$ compared with the average energy acquisition cost of VPP. This coefficient allows penalizing the hours with high energy cost $\left(C_{(i, Y P P)}>C_{\arg (V P P)}\right)$ and benefiting the hours with low energy cost $\left(C_{(i, V P P)}<C_{\text {arg( }(P P)}\right)$. The factor $\alpha$ allows the VPP give more importance to the consumers profile or to the energy acquisition cost. If value of $\alpha_{\text {were equal to zero, the }}$ VPP only considers the consumer's profile.

\section{CASE STUdY}

The present case study uses a Portuguese utility sample of 229 medium voltage (MV) consumers energy consumption measurements, collected throughout an adequate period (3 months in the summer and three months in the winter). The measurements were recorded with a cadence of 15 minutes resulting in 96 values obtained per day. The main goal of this case study is to analyse the impact of the proposed tariffs methodology in contracts of medium voltage consumers aggregated to the VPP.

A clustering methodology proposed in [5] is used to identify the typical profiles of the MV consumers in the winter, in the summer, in workdays and in the weekends. During the pre-processing phase, an artificial neural net (multi-layer perception) was implemented in order to fill out missing values which were identified.

After the pre-processing step, the representative load diagrams have been obtained by averaging the daily load diagrams of each customer. Thus, each customer is represented by just one typical load curve. The obtained representative load profile regards the power consumption, which means that the diagram shape is directly proportional to the amount of the power energy. As it is intended to compare the consumption pattern among customers, and not the consumption values, the power consumption was normalized to the $[0,1]$ range, using the peak power of the each representative load diagram as normalization factor, maintaining the information related to the initial load profile shape. The representative load diagrams of all customers have been separated into two loading regimes, workdays and weekends.

Figure 5 shows the obtained consumers' profiles in this example, for workdays in the winter season. Each curve represents the load profile of the corresponding customer's class. Therefore, each cluster represents a set of clusters with the same consumption pattern. The details regarding the choice of the best partition (best cluster algorithm), and also the cluster validity indices values can be found in $[5,11]$.

\section{Work days}

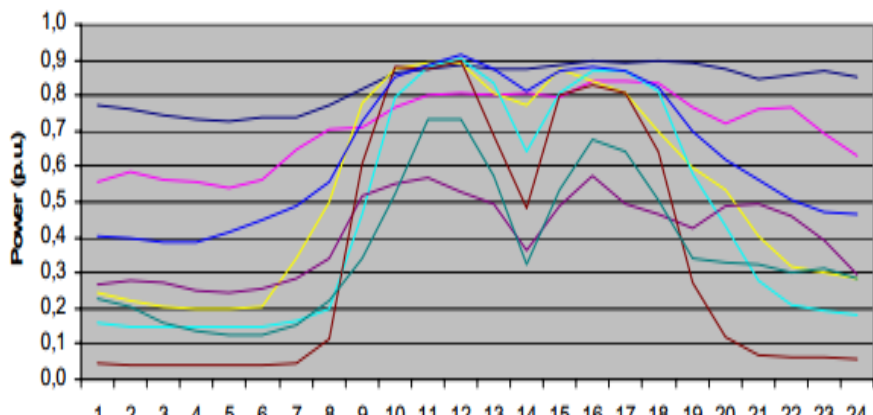

$\begin{array}{llllllllllllllllllllllll}1 & 2 & 3 & 4 & 5 & 6 & 7 & 8 & 9 & 10 & 11 & 12 & 13 & 14 & 15 & 16 & 17 & 18 & 19 & 20 & 21 & 22 & 23 & 24\end{array}$

Time (h)

Figure 5. Consumers profiles in work days

For each one of the obtained profiles the proposed tariffs methodology is applied, considering the number of hours in each TOD period (see Table I). This case study considers that the VPP has a profit of $100 \%$ comparing to the investment in the acquisition of energy on the electricity market.

Figures 6 show the obtained tariffs for global consumer's profiles (consider all consumers collected data) and Figures 7 to 15 shows the obtained tariffs for each consumer profile for work days in winter seasons.

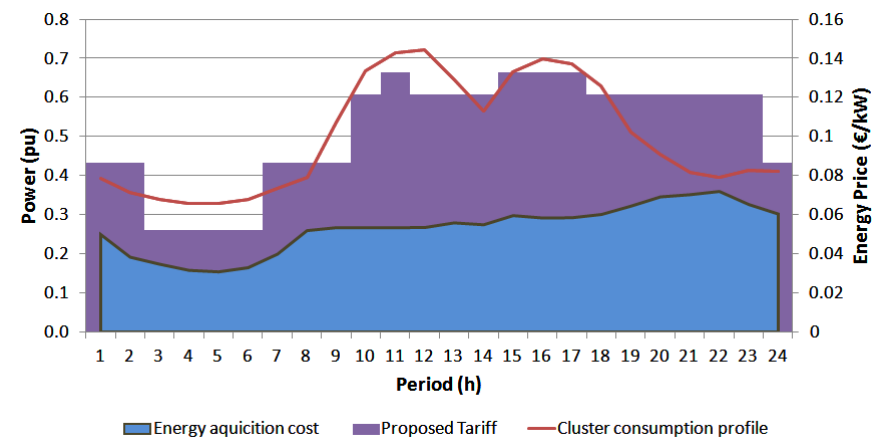

Figure 6. Global profileTarif 


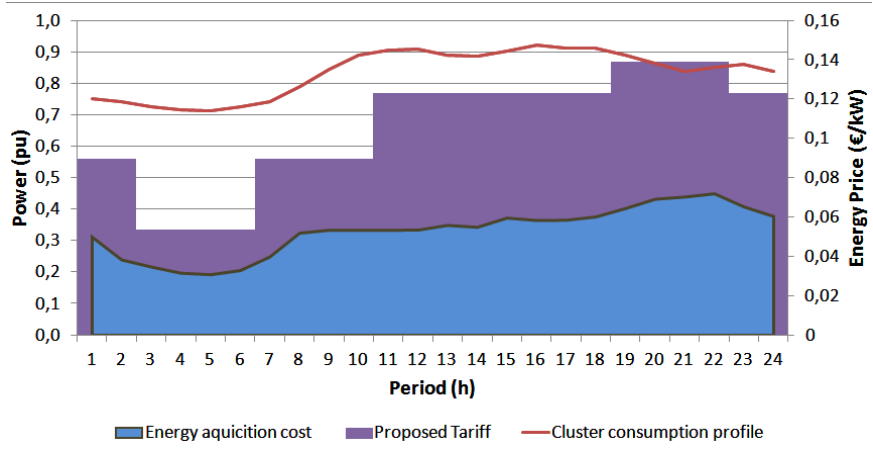

Figure 7. Cluster 1 Tariff for winter session and week days

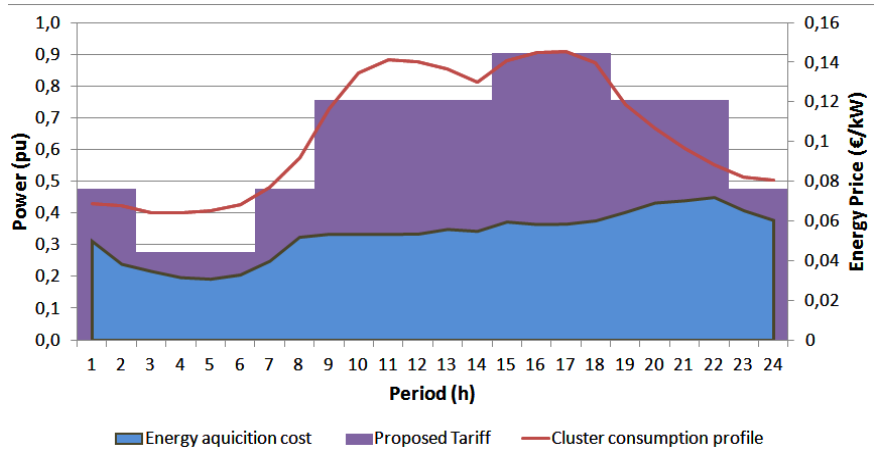

Figure 8. Cluster 2 Tariff for winter session and week days

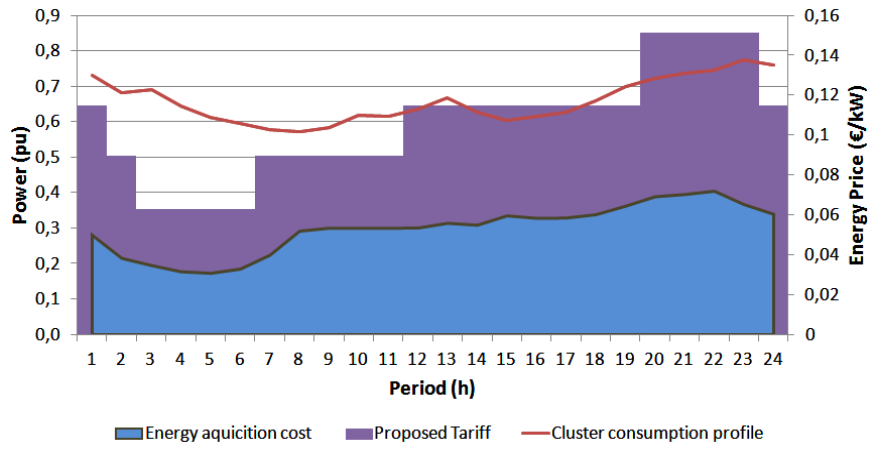

Figure 9. Cluster 3 Tariff for winter session and week days

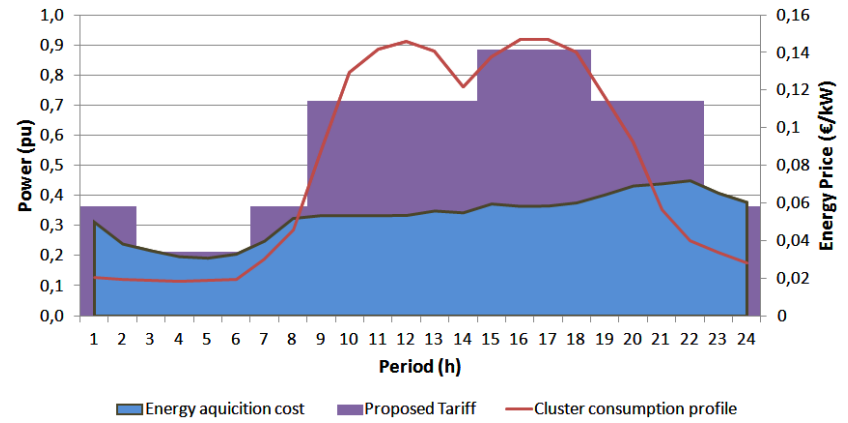

Figure 10. Cluster 4 Tariff for winter session and week days

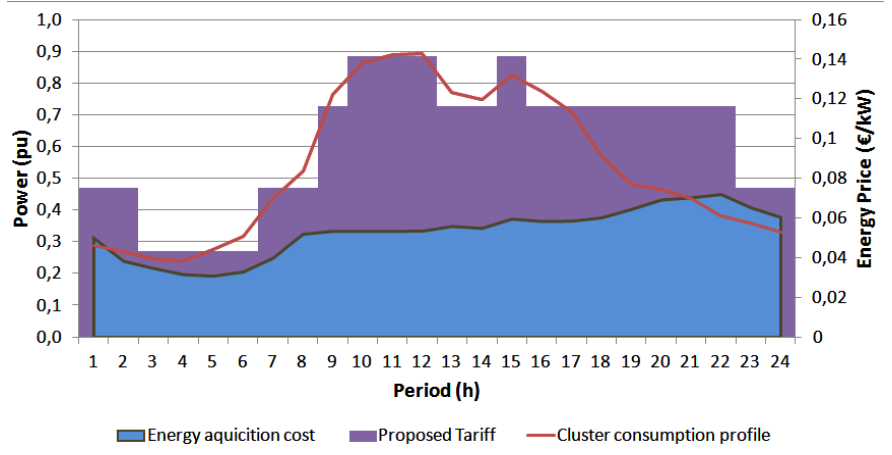

Figure 11. Cluster 5 Tariff for winter session and week days

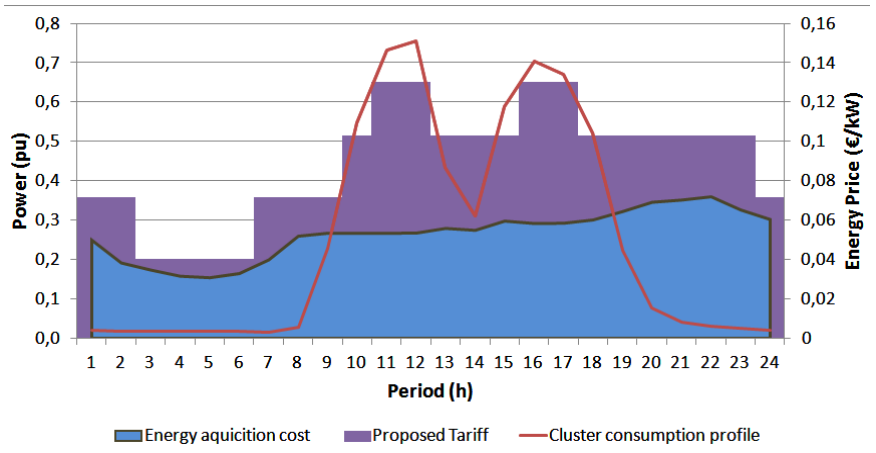

Figure 12. Cluster 6 Tariff for winter session and week days

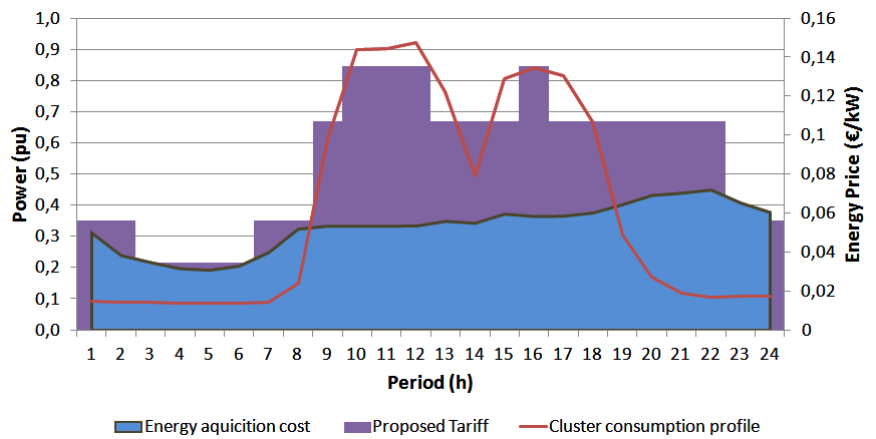

Figure 13. Cluster 7 Tariff for winter session and week days

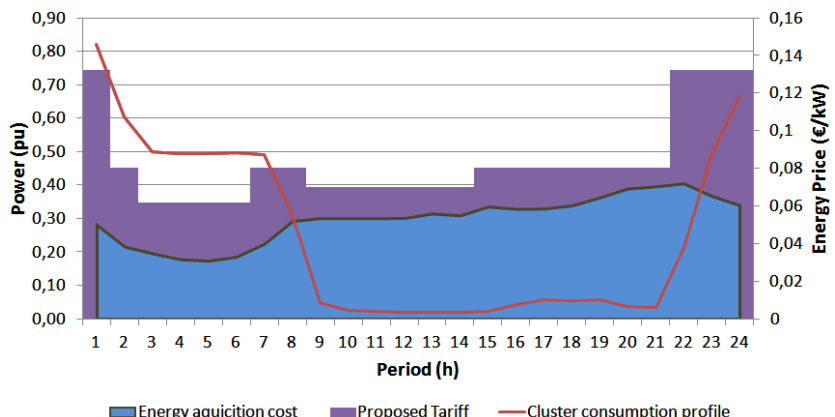

$\square$ Energy aquicition cost $\quad$ Proposed Tariff —Cluster consumption profile

Figure 14. Cluster 8 Tariff for winter session and week days 


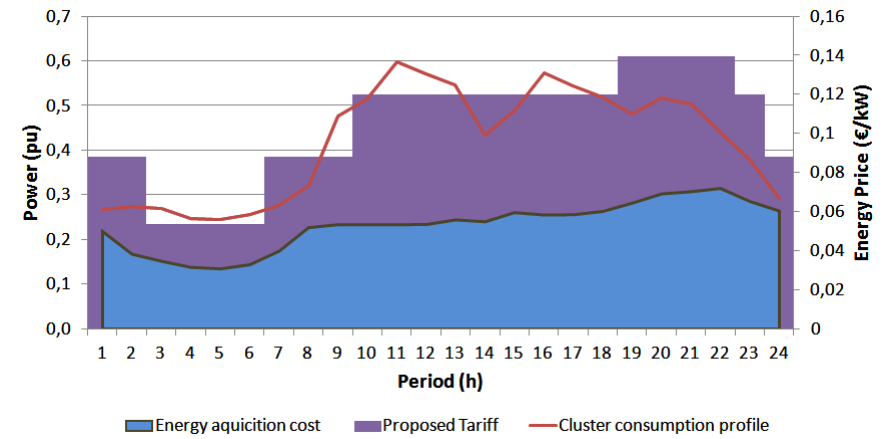

Figure 15. Cluster 9 Tariff for winter session and week days

The considered market price is based in the real prices in MIBEL (Iberian Electricity Market) during the year of 2011 [14] and includes all the taxes and services rates (networks use, reserves guarantee, ancillary services, etc). In the graphics the blue color represents the cost of energy acquisition in the electricity market and the red color represents the energy payments of the consumers. The difference between the areas is the gross profit of the utility.

In Figures 6 to 15 is possible to see high diversity of the proposed tariffs for each consumer profile. In this way each consumer can adapt their consumption for the hours of cheaper energy but this change not penalizes the VPP. For example, in Cluster 2, the consumption profile has two peak hours. However, the VPP only penalize the peak hours with higher energy acquisition cost.

\section{CONCLUSIONS}

In this paper is proposed a tariffs methodology to use by a Virtual Power Player (VPP). The proposed methodology considers the consumption profile of each consumer and adapts the tariffs to each one. Beyond the consumption profiles, the proposed methodology considers also the VPP cost to the acquisition of electricity energy. VPP can balance the importance of the consumption profile and the energy acquisition cost according to their strategy. The regulatory concerns is also considered, namely in the definition of number of hours in each TOD period.

A case study considering 229 real Portuguese consumers and the average MIBEL market clearing price on the year 2011 was presented. In this case study were considered 9 clusters and the tariffs for each one were presented.

\section{REFERENCES}

[1] X. Fang, S. Misra, X. Guoliang, D. Yang, "Smart Grid - The New and Improved Power Grid: A Survey", IEEE Communications Surveys \& Tutorials, vol.14, no.4, pp.944-980, Fourth Quarter 2012

[2] P. Faria, Z. Vale, "Demand response in electrical energy supply: An optimal real time pricing approach", Energy, vol. 36, pp. 5374-5384, August 2011

[3] H. Morais, T. Pinto, Z. Vale, I. Praça, "Multi-Level Negotiation in Smart Grids for VPP Management of Distributed Resources", IEEE Intelligent Systems magazine, Special Issue "Sustainable Energy and Distributed AI", vol. 27, no. 6, pp. 8-16, November-December, 2012

[4] I. Monedero, F. Biscarri, C. León, J. Biscarri, R. Millán, "Midas: Detection of non-technical losses in electrical consumption using neural networks and statistical techniques", International Conference Computational Science and Applications, vol. 3984, Springer Lecture Notes, Computational Science, 2006

[5] S. Ramos, J. Duarte, J. Soares, Z. Vale, F. Duarte, "Typical Load Profiles in the Smart Grid Context - A Clustering Methods Comparison", IEEE Power and Energy Society General Meeting 2012, San Diego, California, USA, 22 - 26 July, 2012

[6] V. Figueiredo, F. Rodrigues, Z. Vale, J. Gouveia, "An Electric Energy Consumer Characterization Framework based on Data Mining Techniques", IEEE Transactions on Power Systems, Vol. 20, pp. 596-602, 2005

[7] T. Pinto, H. Morais, P. Oliveira, Z. Vale, I. Praça, C. Ramos, "A new approach for multi-agent coalition formation and management in the scope of electricity markets", Energy, Vol. 36, Issue 8. pp. 5004-5015, Elsevier, August de 2011

[8] U. Fayyad, G. Piatetsky-Shapiro, P.J. Smyth, R. Uthurasamy, "From Data Mining to Knowledge Discovery: An Overview". In Advances in Knowledge Discovery and Data Mining, pp 1-34, AAAI/MIT Press, 1996

[9] Anil K. Jain, "Data clustering: 50 years beyond K-means". In Pattern Recognition. Letters 31, 2009

[10] J. Frawley, G. Piatetsky-Shapiro, C. Matheus, "Knowledge Discovery in Databases: An Overview", Technical Report, 1995

[11] S. Ramos, Z. Vale, "Data Mining techniques application in Power Distribution Utilities", IEEE PES Transmission and Distribution Conference and Exposition, Chicago, Illinois, USA, 21-24 April, 2008

[12] T. Zhang, G. Zhang, J. Lu, X. Feng, W. Yang, "A New Index and Classification Approach for Load Pattern Analysis of Large Electricity Customers", IEEE Transactions on Power Systems, vol.27, no.1, pp.153-160, Feb. 2012.

[13] I. Apolinário, N. Felizardo, A. Leite Garcia, P. Oliveira, A. Trindade, P. Verdelho, "Determination of Time-Of-Day Schedules in the Portuguese Electric Sector", IEEE Power Engineering Society General Meeting, Montreal, Quebec, Canada, 18-22 June, 2006

[14] Mercado Ibérico de Eléctricidade, Accessed online: www.mibel.com 